\title{
San Francisco Bay Triennial Bird Egg Monitoring Program for Contaminants, California-2018
}

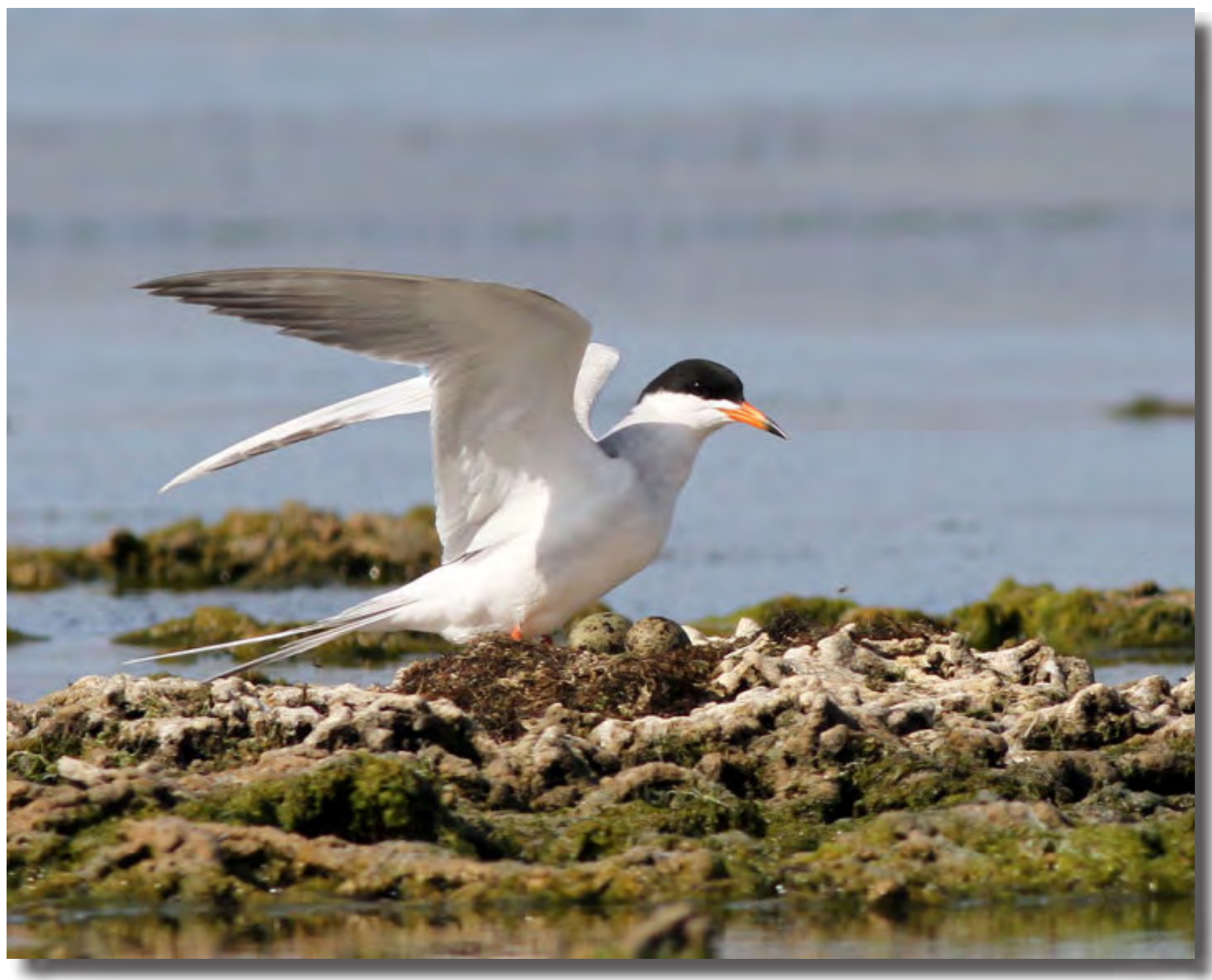

\section{Data Series 1114}

U.S. Department of the Interior

U.S. Geological Survey 
Cover photograph: Forster's tern standing over nest and eggs. Photograph by Abe Borker, University of California, Santa Cruz, graduate student collaborating with U.S. Geological Survey at time of photo, July 9, 2010. 


\section{San Francisco Bay Triennial Bird Egg Monitoring Program for Contaminants, California-2018}

By Joshua T. Ackerman, C. Alex Hartman, Mark P. Herzog, and Matthew Toney

Data Series 1114 


\title{
U.S. Department of the Interior \\ DAVID BERNHARDT, Secretary
}

\author{
U.S. Geological Survey \\ James F. Reilly II, Director
}

U.S. Geological Survey, Reston, Virginia: 2019

For more information on the USGS - the Federal source for science about the Earth, its natural and living resources, natural hazards, and the environment-visit https://www.usgs.gov or call 1-888-ASK-USGS.

For an overview of USGS information products, including maps, imagery, and publications,

visit https://store.usgs.gov.

Any use of trade, firm, or product names is for descriptive purposes only and does not imply endorsement by the U.S. Government.

Although this information product, for the most part, is in the public domain, it also may contain copyrighted materials as noted in the text. Permission to reproduce copyrighted items must be secured from the copyright owner.

Suggested citation:

Ackerman, J.T., Hartman, C.A., Herzog, M.P., and Toney, M., 2019, San Francisco Bay triennial bird egg monitoring program for contaminants—2018: U.S. Geological Survey Data Series 1114, 14 p., https://doi.org/10.3133/ds1114. 


\section{Acknowledgments}

We thank the San Francisco Bay Regional Monitoring Program and San Francisco Estuary Institute for funding and project support and U.S. Fish and Wild life Service Don Edwards San

Francisco Bay National Wildlife Refuge, California Department of Transportation, and Black Mallard Duck Club for logistical support in collecting samples. We also thank Breanne Cooney, Jeanne Fasan, Tyler Glaser, and Budget Tree Service for assistance in the field. 


\section{Contents}

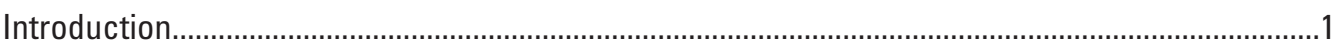

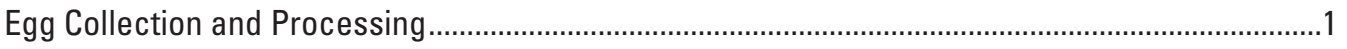

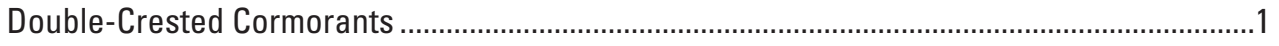

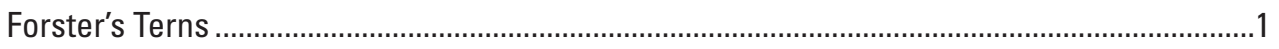

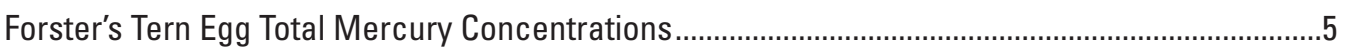

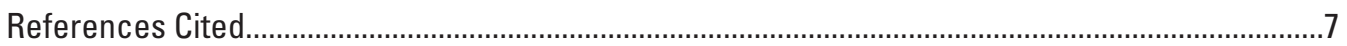

Appendix 1. Double-Crested Cormorant Egg Collection and Measurement Data for Eggs Collected by U.S. Geological Survey From San Francisco Bay, California, in 2018...............8

Appendix 2. Quality Assurance and Quality Control Results for Total Mercury Analyses of Forster's Tern Eggs Collected by U.S. Geological Survey from San Francisco Bay, California, in 2018.

Appendix 3. Forster's Tern Egg Collection Data and Total Mercury Concentrations for Eggs Collected by USGS From San Francisco Bay, California, in 2018 .......................................12

\section{Figures}

1. Map showing double-crested cormorant colony locations that were sampled by U.S. Geological Survey for the Regional Monitoring Program for Water Quality in San Francisco Bay, California, in 2018

2. Map showing Forster's tern colony locations that were sampled by U.S. Geological Survey for the Regional Monitoring Program for Water Quality in San Francisco Bay, California, in 2018

3. Bar graph showing total mercury concentrations in Forster's tern eggs sampled from four colonies in San Francisco Bay during the 2018 breeding season

4. Graph showing mean \pm standard error total mercury concentrations in Forster's tern eggs in 2009 


\section{Conversion Factors}

International System of Units to U.S. customary units

\begin{tabular}{|c|c|c|}
\hline Multiply & By & To obtain \\
\hline \multicolumn{3}{|c|}{ Length } \\
\hline millimeter (mm) & 0.03937 & inch (in.) \\
\hline \multicolumn{3}{|c|}{ Area } \\
\hline square meter $\left(\mathrm{m}^{2}\right)$ & 0.0002471 & acre \\
\hline hectare (ha) & 2.471 & acre \\
\hline square hectometer $\left(\mathrm{hm}^{2}\right)$ & 2.471 & acre \\
\hline square kilometer $\left(\mathrm{km}^{2}\right)$ & 247.1 & acre \\
\hline square centimeter $\left(\mathrm{cm}^{2}\right)$ & 0.001076 & square foot $\left(\mathrm{ft}^{2}\right)$ \\
\hline square meter $\left(\mathrm{m}^{2}\right)$ & 10.76 & square foot $\left(\mathrm{ft}^{2}\right)$ \\
\hline square centimeter $\left(\mathrm{cm}^{2}\right)$ & 0.1550 & square inch $\left(\mathrm{ft}^{2}\right)$ \\
\hline square hectometer $\left(\mathrm{hm}^{2}\right)$ & 0.003861 & section ( 640 acres or 1 square mile) \\
\hline hectare (ha) & 0.003861 & square mile $\left(\mathrm{mi}^{2}\right)$ \\
\hline square kilometer $\left(\mathrm{km}^{2}\right)$ & 0.3861 & square mile $\left(\mathrm{mi}^{2}\right)$ \\
\hline \multicolumn{3}{|c|}{ Volume } \\
\hline milliliter $(\mathrm{mL})$ & 0.033814 & ounce, fluid (fl. oz) \\
\hline \multicolumn{3}{|c|}{ Mass } \\
\hline $\operatorname{gram}(\mathrm{g})$ & 0.03527 & ounce, avoirdupois (oz) \\
\hline
\end{tabular}

Temperature in degrees Celsius $\left({ }^{\circ} \mathrm{C}\right)$ may be converted to degrees Fahrenheit $\left({ }^{\circ} \mathrm{F}\right)$ as ${ }^{\circ} \mathrm{F}=\left(1.8 \times{ }^{\circ} \mathrm{C}\right)+32$. 


\title{
Datum
}

Horizontal coordinate information is referenced to the North American Datum of 1983 (NAD 83).

\section{Supplemental Information}

Concentrations of total mercury $(\mathrm{THg})$ in eggs are given in micrograms per gram $(\mu \mathrm{g} / \mathrm{g})$.

\author{
Abbreviations \\ fww fresh wet weight \\ RMP Regional Monitoring Program for Water Quality in San Francisco Bay \\ SFEI San Francisco Estuary Institute \\ THg total mercury \\ USGS U.S. Geological Survey
}




\title{
San Francisco Bay Triennial Bird Egg Monitoring Program for Contaminants, California—2018
}

\author{
By Joshua T. Ackerman, C. Alex Hartman, Mark P. Herzog, and Matthew Toney
}

\section{Introduction}

The Regional Monitoring Program for Water Quality in San Francisco Bay (RMP), administered by the San Francisco Estuary Institute, is a large-scale effort to monitor contaminant trends in water, sediment, fish, and birds throughout San Francisco Bay (San Francisco Estuary Institute, 2016). As part of the RMP and the U.S. Geological Survey (USGS) long-term Wildlife Contaminants Program, the USGS samples doublecrested cormorant (Phalacrocorax auritus) and Forster's tern (Sterna forsteri) eggs throughout the San Francisco Bay approximately every 3 years to assess temporal trends in contaminant concentrations. This sampling has previously been carried out by USGS in 2009, 2012, and 2016. This document summarizes egg collections for 2018, as well as mercury concentrations in Forster's tern eggs on an individual egg basis. These data are available in a USGS data release (Ackerman and others, 2019).

\section{Egg Collection and Processing}

\section{Double-Crested Cormorants}

Double-crested cormorant eggs were sampled between May 10 and 16, 2018, from three locations: (1) Wheeler Island, (2) Richmond-San Rafael Bridge, and (3) Pond A5/ A7 levee in the eastern Alviso salt pond complex (region not shown on fig. 1) of south San Francisco Bay (fig. 1). The Pond A5/A7 cormorant egg sampling location replaced the nearby A9/A10 collection site used for RMP in 2006, 2012, and 2016 because cormorants did not nest at A9/A10 in 2018. A total of 21 eggs were collected from 21 separate nests from each of the Richmond-San Rafael Bridge and Pond A5/A7 levee locations (total of 42 eggs). At the Wheeler Island location, doublecrested cormorants nest in dying Eucalyptus trees (Eucalyptus globulus) on private land. Because of difficulties with site access owing to a private levee road inhibiting use of a boom truck until a later date in the nesting season and difficult climbing conditions of the dying Eucalyptus trees (for the tree climbers externally hired by San Francisco Estuary Institute;
SFEI), we collected six eggs from three nests (four eggs from one nest, one egg from each of two nests) at the Wheeler Island location. We measured egg mass, egg length, and egg width for each double-crested cormorant egg (appendix 1; Ackerman and others, 2019), and then the double-crested cormorant eggs were shipped unopened in a highly padded package to SGS AXYS (Sidney, British Columbia, Canada) on July 9, 2018, for dissection, processing, contaminant analyses, and further reporting. AXYS confirmed receiving all 48 eggs on July 13, 2018, and AXYS reported that 11 of the 48 eggs were cracked during shipment with some contents released into the surrounding Whirl-Pak bag. The remaining 37 eggs were all intact.

\section{Forster's Terns}

Forster's tern eggs were sampled between May 17 and July 17, 2018, from four different colonies: (1) Pond A3W, (2) Pond N1, (3) New Chicago Marsh, and (4) Pond SF2 at the Don Edwards San Francisco Bay National Wildlife Refuge (fig. 2; refuge not shown in fig. 2). Ponds A3W, N1, and SF2 replaced sampling locations used in previous years (for example, Ponds A1, A2W, A7, AB1, and AB2; ponds not shown in fig. 2) because Forster's terns either did not nest at these sites in 2018 or did not nest in sufficient numbers to allow for egg collections. At another location used in previous years, Hayward Shoreline Regional Park (area not shown in fig. 2), site managers did not allow egg collections in 2018.

A total of 21 Forster's tern eggs were collected from 21 separate nests at each of the 4 locations (total of 84 eggs). Eggs were collected randomly from monitored nests at early incubation stages determined via egg flotation (Ackerman and Eagles-Smith, 2010). Eggs were placed in egg cartons and stored on wet ice until transport back to the laboratory, where they were stored in a refrigerator until dissection. During egg dissection, refrigerated eggs were allowed to warm to room temperature before egg length and width were measured to the nearest 0.01 millimeter $(\mathrm{mm})$ using digital calipers (Fowler High Precision), and total egg weight (including eggshell) was weighed to the nearest 0.01 gram (g) on a digital balance (Ohaus Adventurer Pro, model AV212C; 
Ohaus). Using clean, stainless steel instruments, we cut a hole approximately $15 \mathrm{~mm}$ in diameter in the wide end of each egg and removed the entire contents into a chemically-cleaned and certified 60-milliliter $(\mathrm{mL})$ jar $\left(\right.$ Thermo Scientific ${ }^{\mathrm{TM}}$ Wide-Mouth Short-Profile Amber Glass Jars, with PTFE-lined polypropylene lid). Egg content (without eggshell) was then weighed with a digital balance to the nearest $0.01 \mathrm{~g}$, and egg contents were stored at $-20{ }^{\circ} \mathrm{C}$ until processing and mercury determination. During processing, eggs were thawed at room temperature, and then the entire egg contents were dried at $50{ }^{\circ} \mathrm{C}$ for $>96$ hours until completely dried. To determine moisture content, we reweighed dried egg contents with a digital balance to the nearest $0.0001 \mathrm{~g}$ (Ohaus Adventurer Balance, model AR064; Ohaus). Dried egg contents were then homogenized to a powder using a spice grinder with stainless steel blades, followed by further grinding by hand in a mortar and pestle. Processed egg samples were stored in a desiccator until mercury determination.

Each Forster's tern egg was analyzed for total mercury (THg) concentrations at the USGS Dixon Field Station Environmental Mercury Laboratory on a Nippon MA-3000 Direct Mercury Analyzer (Nippon Instruments North America, College Station, Texas, U.S.A.), following Environmental Protection Agency Method 7473 (U.S. Environmental Protection Agency, 2000), using an integrated sequence of drying, thermal decomposition, catalytic conversion, and then amalgamation, followed by atomic absorption spectroscopy. Prior research has demonstrated that an average of 96 percent of the mercury in eggs is in the methylmercury form and that total mercury concentrations in eggs are highly correlated with methylmercury concentrations in eggs (Ackerman and others, 2013b). We converted the dry weight THg concentrations for Forster's tern egg contents to a fresh wet weight (fww) THg concentration for each individual egg's contents, following the methods of Ackerman and others (2013b) and accounting for the thickness of the eggshell following Herzog and others (2016).

Quality assurance measures included analysis of a certified reference material (dogfish muscle tissue [DORM] and lobster hepatopancreas [TORT] certified by the National Research Council of Canada, Ottawa, Canada), system blank, method blank, continuing calibration verification, and duplicate with each set of approximately 10 samples and 2 spiked duplicates with each set of approximately 20 samples. In total, quality assurance measures for the 84 Forster's tern eggs included 10 system blanks, 10 method blanks, 10 continuing calibration verification, 13 certified reference material, 10 duplicates, 10 matrix spikes, and 5 matrix spike duplicates. Recoveries (mean \pm standard deviation) were $101.3 \pm 2.7$ percent $(n=13)$ for certified reference materials, $100.3 \pm 1.9$ percent $(n=10)$ for continuing calibration verifications, and $100.0 \pm 1.8$ percent $(n=10)$ for matrix spikes. Relative percent difference averaged $2.0 \pm 1.6$ percent $(\mathrm{n}=10)$ for duplicates and $1.6 \pm 1.3$ percent $(\mathrm{n}=5)$ for matrix spike duplicates (appendix 2; Ackerman and others, 2019).

After homogenizing the eggs, equal masses (dried) from each of seven randomly chosen eggs per colony were combined to make three separate composite samples of seven eggs each per Forster's tern colony (appendix 2; Ackerman and others, 2019). Each composite sample was then re-homogenized, and aliquots were put into jars provided by each external lab. Forster's tern egg composite samples were shipped at room temperature to the California Department of Fish and Wildlife Moss Landing Marine Lab (Moss Landing, California) on December 10, 2018, for selenium determination and further reporting and shipped at room temperature to AXYS Analytical laboratories on February 19, 2019, for polybrominated diphenyl ethers (PBDE) analyses and further reporting. 


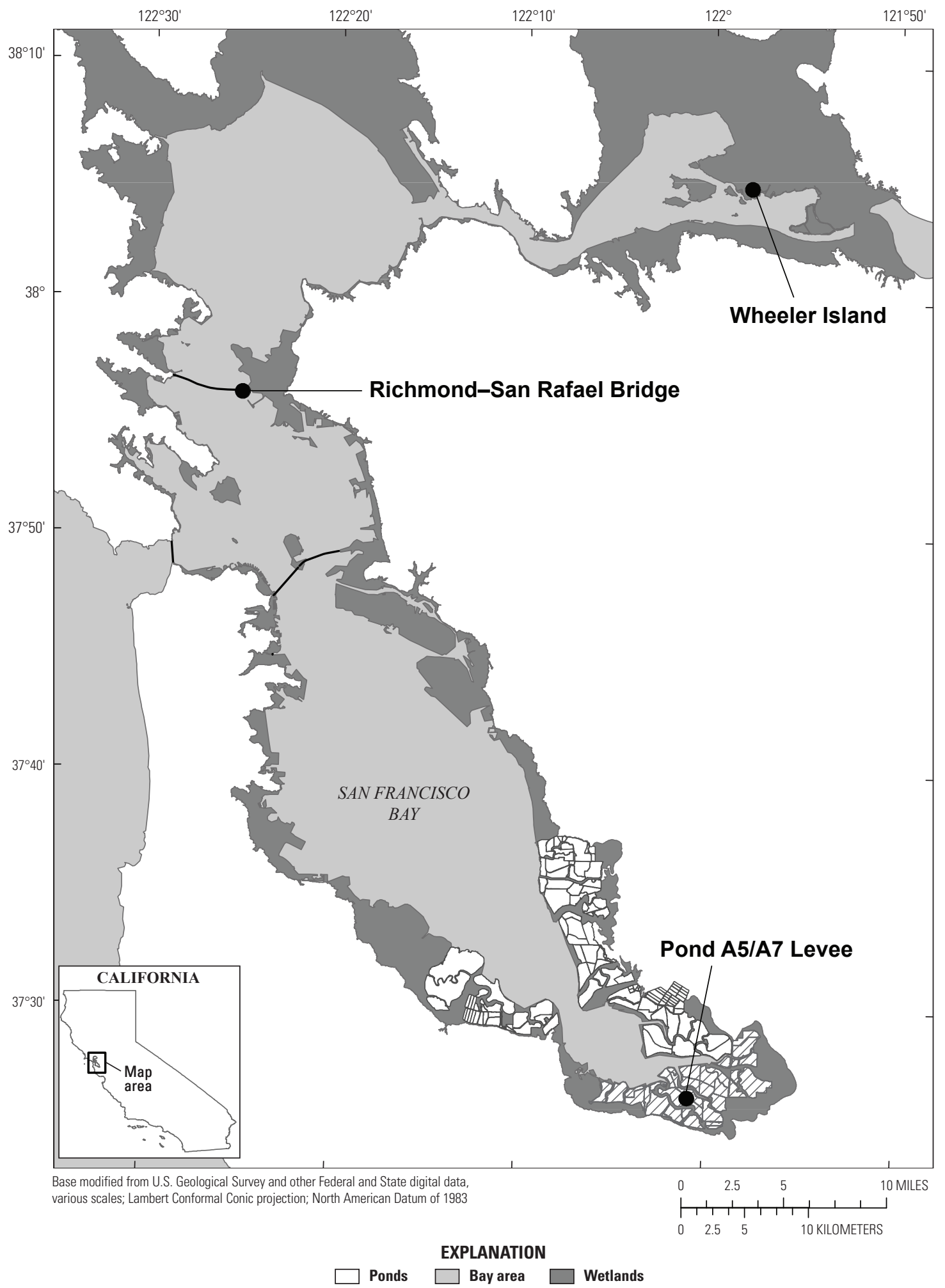

Figure 1. Double-crested cormorant colony locations that were sampled by U.S. Geological Survey for the Regional Monitoring Program for Water Quality in San Francisco Bay, California, in 2018. 


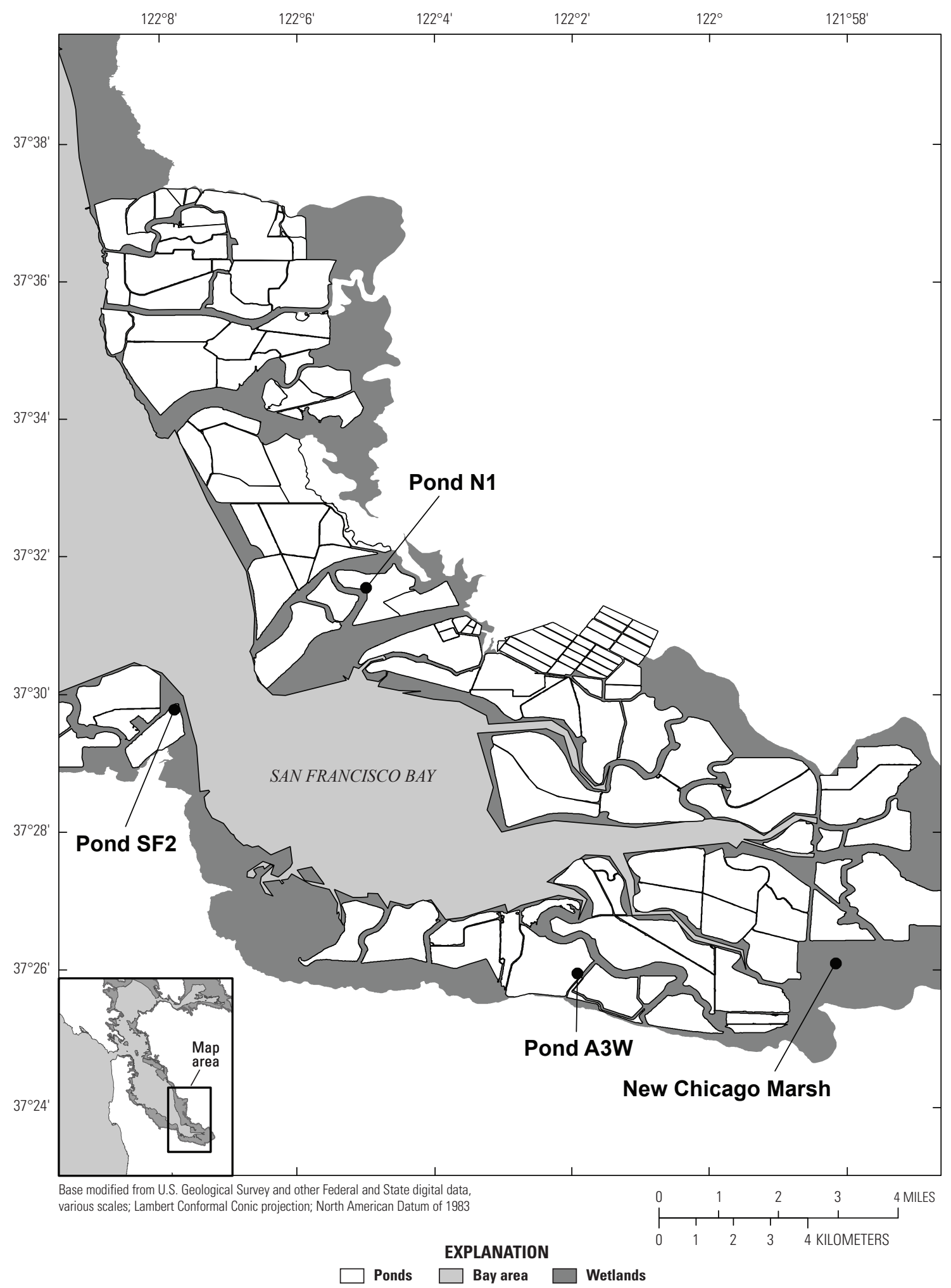

Figure 2. Forster's tern colony locations that were sampled by U.S. Geological Survey for the Regional Monitoring Program for Water Quality in San Francisco Bay, California, in 2018. 


\section{Forster's Tern Egg Total Mercury Concentrations}

Across all sites, the geometric mean ( \pm standard error) THg concentrations in Forster's tern eggs was $1.3 \pm 0.07$ micrograms per gram $(\mu \mathrm{g} / \mathrm{g}) \mathrm{fww}$, and concentrations in individual eggs ranged from $0.30 \mu \mathrm{g} / \mathrm{g}$ to $3.60 \mu \mathrm{g} / \mathrm{g}$ fww (appendix 3; Ackerman and others, 2019). Mercury concentrations in Forster's tern eggs varied slightly among colonies (ANOVA: $\mathrm{F}_{3,80}=2.52, \mathrm{P}=0.06$; fig. 3 ). Egg mercury concentrations varied greatly among individuals within the same colony (fig. 3), indicating that using composite samples would not adequately characterize risk of mercury to Forster's terns in San Francisco Bay.

We evaluated risk to Forster's tern breeding productivity by assessing individual egg mercury concentrations in relation to a benchmark value of $0.75 \mu \mathrm{g} / \mathrm{g}$ fww, which is associated with Forster's terns beginning the process of methylmercury demethylation in the liver (EaglesSmith and others, 2009; Ackerman and others, 2016a). Overall, 85 percent of eggs sampled (71 of 84) exceeded this $0.75 \mu \mathrm{g} / \mathrm{g}$ fww benchmark. On a site-specific basis, 100 percent of eggs at the Pond A $3 \mathrm{~W}$ colony, 76 percent of eggs at the New Chicago Marsh colony, 86 percent of eggs at the Pond SF2 colony, and 76 percent of eggs at the Pond N1 colony exceeded $0.75 \mu \mathrm{g} / \mathrm{g}$ fww. Geometric mean mercury concentrations in Forster's tern eggs were, on average, higher in $2018(1.30 \pm 0.07 \mu \mathrm{g} / \mathrm{g}$ fww $)$ compared to 2016 $(1.07 \pm 0.07 \mu \mathrm{g} / \mathrm{g}$ fww), $2012(1.09 \pm 0.04 \mu \mathrm{g} / \mathrm{g}$ fww), and 2009 (0.97 $\pm 0.05 \mu \mathrm{g} / \mathrm{g}$ fww) (fig. 4). The samples document egg mercury concentrations that continue to be above benchmarks for high risk of impaired reproduction to Forster's terns in San Francisco Bay.

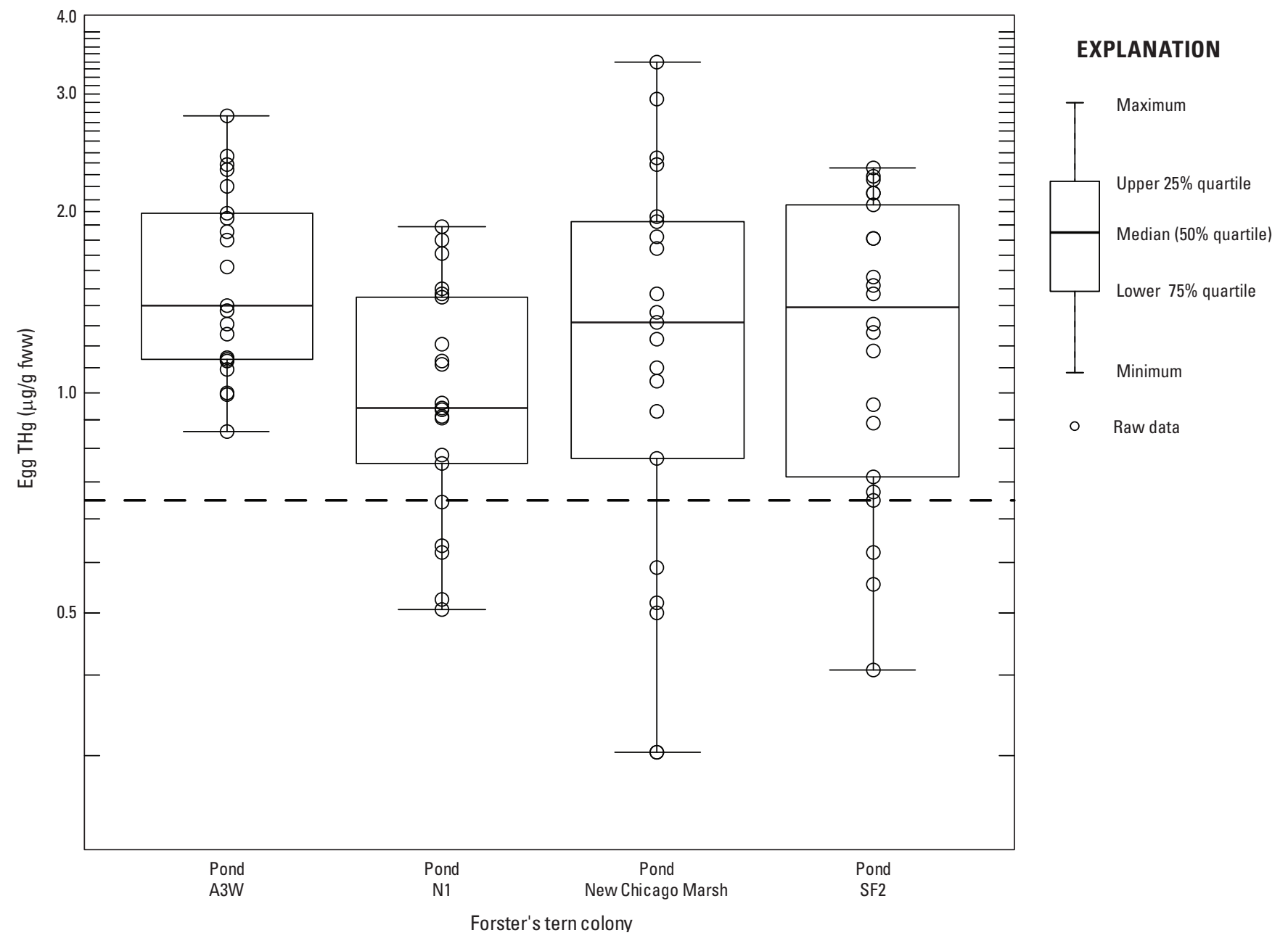

Figure 3. Total mercury (THg) concentrations (micrograms per gram [ $\mu \mathrm{g} / \mathrm{g}$ ] fresh wet weight [fww]) in Forster's tern eggs sampled from four colonies in San Francisco Bay during the 2018 breeding season. The stippled line indicates a benchmark value of $0.75 \mu \mathrm{g} / \mathrm{g}$ fww where Forster's tern health may be impaired (Eagles-Smith and others, 2009; Ackerman and others, 2016a). 


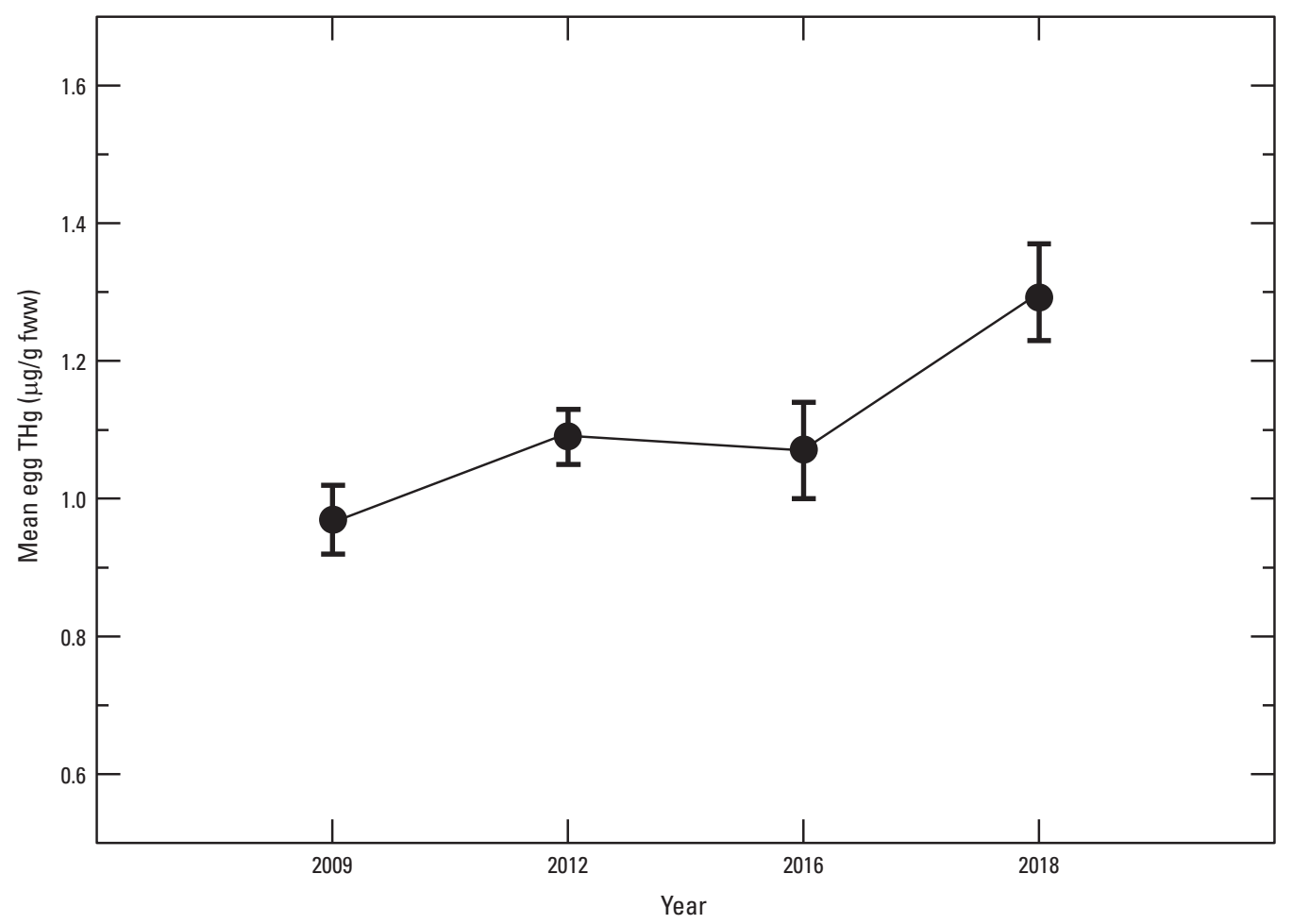

Figure 4. Mean \pm standard error total mercury ( $\mathrm{THg}$ ) concentrations (micrograms per gram [ $\mu \mathrm{g} / \mathrm{g}$ ] fresh wet weight [fww]) in Forster's tern eggs in 2009 (Eagles-Smith and Ackerman, 2009), 2012 (Ackerman and others, 2013a), 2016 (Ackerman and others, 2016b), and 2018 (this report). 


\section{References Cited}

Ackerman, J.T., and Eagles-Smith, C.A., 2010, Accuracy of egg flotation throughout incubation to determine embryo age and incubation day in waterbird nests: The Condor, v. 112 , no. 3, p. 438-446, https://doi.org/10.1525/cond.2010.090070.

Ackerman, J.T., Eagles-Smith, C.A., Herzog, M.P., and Hartman, C.A., 2016a, Maternal transfer of contaminants in birds-Mercury and selenium concentrations in parents and their eggs: Environmental Pollution, v. 210, p. 145-154, https://doi.org/10.1016/j.envpol.2015.12.016.

Ackerman, J.T., Hartman, C.A., Herzog, M.P., and Toney, M., 2016b, San Francisco Bay triennial bird egg monitoring program for contaminants - 2016 draft data summary: Dixon, Calif., U.S. Geological Survey, Western Ecological Research Center, $19 \mathrm{p}$.

Ackerman, J.T., Hartman, A., Herzog, M., and Toney, M., 2019, USGS Regional Monitoring Program bird egg data (CEDEN): U.S. Geological Survey data release, accessed March 2019 at https://usgs.ornl.gov/metadata/catalog/xml/ Western_Ecological_Research_Center/66fe374a-b955-450f9042-7988c1fa5c8b.xml.

Ackerman, J.T., Herzog, M.P., and Hartman, C.A., 2013a, San Francisco Bay triennial bird egg monitoring program for contaminants - 2012 draft data summary: Davis, Calif., U.S. Geological Survey, Western Ecological Research Center, $18 \mathrm{p}$.
Ackerman, J.T., Herzog, M.P., and Schwarzbach, S.E., 2013b, Methylmercury is the predominant form of mercury in bird eggs-A synthesis: Environmental Science \& Technology, v. 47, no. 4, p. 2052-2060, https://doi.org/10.1021/es304385y.

Eagles-Smith, C.A., and Ackerman, J.T., 2009, San Francisco Bay triennial bird egg monitoring program for contaminants - 2009 draft data summary: Davis, Calif., U.S. Geological Survey, Western Ecological Research Center, $10 \mathrm{p}$.

Eagles-Smith, C.A., Ackerman, J.T., Yee, J., and Adelsbach, T.L., 2009, Mercury demethylation in waterbird livers-Dose-response thresholds and differences among species: Environmental Toxicology and Chemistry, v. 28, no. 3, p. 568-577, https://doi.org/10.1897/08-245.1.

Herzog, M.P., Ackerman, J.T., Eagles-Smith, C.A., and Hartman, C.A., 2016, It's what's inside that countsEgg contaminant concentrations are influenced by estimates of egg density, egg volume, and fresh egg mass: Ecotoxicology (London, England), v. 25, no. 4, p. 770-776, https://doi.org/10.1007/s10646-016-1635-9.

San Francisco Estuary Institute, 2016, 2015 annual monitoring report. The Regional Monitoring Program for Water Quality in the San Francisco Bay (RMP): Richmond, Calif., San Francisco Estuary Institute, Contribution No. 775, 57 p.

U.S. Environmental Protection Agency, 2000, Method 7473, Mercury in solids and solutions by thermal decomposition, amalgamation, and atomic absorption spectrophotometry, in Test methods for evaluating solid waste, physical/ chemical methods; SW 846, Update IVA: Washington, D.C., U.S. Government Printing Office, 17 p. 


\section{Appendix 1. Double-Crested Cormorant Egg Collection and Measurement Data for Eggs Collected by U.S. Geological Survey (USGS) From San Francisco Bay, California, in 2018}

[g, gram; ID, identification; mm, millimeter; mm/dd/yyyy, month/day/year; NAD 83, North American Datum of 1983; UTM, Universal Transverse Mercator coordinate system; USGS, U.S. Geological Survey]

\begin{tabular}{|c|c|c|c|c|c|c|c|c|c|c|c|}
\hline $\begin{array}{l}\text { USGS } \\
\text { egg ID }\end{array}$ & Species & $\begin{array}{c}\text { Collection } \\
\text { date } \\
\text { (mm/dd/yyyy) }\end{array}$ & Year & Location & Station code & $\begin{array}{c}\text { UTM- } \\
\text { easting } \\
\text { (NAD 83) }\end{array}$ & $\begin{array}{c}\text { UTM- } \\
\text { northing } \\
\text { (NAD 83) }\end{array}$ & $\begin{array}{c}\text { USGS egg } \\
\text { measuring date } \\
\text { (mm/dd/yyyy) }\end{array}$ & $\begin{array}{c}\text { USGS whole } \\
\text { egg mass } \\
\text { (g) }\end{array}$ & $\begin{array}{l}\text { USGS whole } \\
\text { egg length } \\
\text { (mm) }\end{array}$ & $\begin{array}{l}\text { USGS whole } \\
\text { egg width } \\
\text { (mm) }\end{array}$ \\
\hline RB-1 & Double-crested cormorant & $05 / 16 / 2018$ & 2018 & Richmond Bridge & 2EEPSRB & 549605 & 4198701 & 06/07/2018 & 43.90 & 59.15 & 38.87 \\
\hline RB-2 & Double-crested cormorant & $05 / 16 / 2018$ & 2018 & Richmond Bridge & 2EEPSRB & 549605 & 4198701 & 06/07/2018 & 41.31 & 62.25 & 37.49 \\
\hline RB-3 & Double-crested cormorant & $05 / 16 / 2018$ & 2018 & Richmond Bridge & 2EEPSRB & 549605 & 4198701 & $06 / 07 / 2018$ & 48.51 & 57.97 & 40.93 \\
\hline RB-4 & Double-crested cormorant & $05 / 16 / 2018$ & 2018 & Richmond Bridge & 2EEPSRB & 549605 & 4198701 & 06/07/2018 & 42.98 & 64.19 & 36.54 \\
\hline RB-5 & Double-crested cormorant & $05 / 16 / 2018$ & 2018 & Richmond Bridge & 2EEPSRB & 549605 & 4198701 & $06 / 07 / 2018$ & 50.01 & 60.67 & 41.37 \\
\hline RB-6 & Double-crested cormorant & $05 / 16 / 2018$ & 2018 & Richmond Bridge & 2EEPSRB & 549605 & 4198701 & $06 / 07 / 2018$ & 42.67 & 55.09 & 39.07 \\
\hline RB-7 & Double-crested cormorant & 05/16/2018 & 2018 & Richmond Bridge & 2EEPSRB & 549605 & 4198701 & $06 / 07 / 2018$ & 50.41 & 65.26 & 39.95 \\
\hline RB-8 & Double-crested cormorant & 05/16/2018 & 2018 & Richmond Bridge & 2EEPSRB & 549605 & 4198701 & $06 / 07 / 2018$ & 41.81 & 56.83 & 38.37 \\
\hline RB-9 & Double-crested cormorant & 05/16/2018 & 2018 & Richmond Bridge & 2EEPSRB & 549605 & 4198701 & $06 / 07 / 2018$ & 45.93 & 57.67 & 39.67 \\
\hline RB-10 & Double-crested cormorant & $05 / 16 / 2018$ & 2018 & Richmond Bridge & 2EEPSRB & 549605 & 4198701 & 06/07/2018 & 39.69 & 55.81 & 38.79 \\
\hline RB-11 & Double-crested cormorant & 05/16/2018 & 2018 & Richmond Bridge & 2EEPSRB & 549605 & 4198701 & $06 / 07 / 2018$ & 49.79 & 60.97 & 40.21 \\
\hline RB-12 & Double-crested cormorant & $05 / 16 / 2018$ & 2018 & Richmond Bridge & 2EEPSRB & 549605 & 4198701 & $06 / 07 / 2018$ & 42.83 & 59.75 & 38.65 \\
\hline RB-13 & Double-crested cormorant & 05/16/2018 & 2018 & Richmond Bridge & 2EEPSRB & 549605 & 4198701 & $06 / 07 / 2018$ & 42.69 & 59.87 & 37.91 \\
\hline RB-14 & Double-crested cormorant & $05 / 16 / 2018$ & 2018 & Richmond Bridge & 2EEPSRB & 549605 & 4198701 & $06 / 07 / 2018$ & 40.75 & 61.73 & 36.13 \\
\hline RB-15 & Double-crested cormorant & $05 / 16 / 2018$ & 2018 & Richmond Bridge & 2EEPSRB & 549605 & 4198701 & $06 / 07 / 2018$ & 46.46 & 58.98 & 39.31 \\
\hline RB-16 & Double-crested cormorant & $05 / 16 / 2018$ & 2018 & Richmond Bridge & 2EEPSRB & 549605 & 4198701 & $06 / 07 / 2018$ & 47.02 & 63.77 & 38.74 \\
\hline RB-17 & Double-crested cormorant & $05 / 16 / 2018$ & 2018 & Richmond Bridge & 2EEPSRB & 549605 & 4198701 & $06 / 07 / 2018$ & 51.12 & 65.14 & 39.18 \\
\hline RB-18 & Double-crested cormorant & 05/16/2018 & 2018 & Richmond Bridge & 2EEPSRB & 549605 & 4198701 & $06 / 07 / 2018$ & 45.86 & 57.69 & 39.98 \\
\hline RB-19 & Double-crested cormorant & 05/16/2018 & 2018 & Richmond Bridge & 2EEPSRB & 549605 & 4198701 & $06 / 07 / 2018$ & 43.19 & 59.43 & 38.29 \\
\hline RB-20 & Double-crested cormorant & $05 / 16 / 2018$ & 2018 & Richmond Bridge & 2EEPSRB & 549605 & 4198701 & $06 / 07 / 2018$ & 57.91 & 61.52 & 42.82 \\
\hline RB-21 & Double-crested cormorant & 05/16/2018 & 2018 & Richmond Bridge & 2EEPSRB & 549605 & 4198701 & $06 / 07 / 2018$ & 56.19 & 66.09 & 40.85 \\
\hline SB-1 & Double-crested cormorant & $05 / 15 / 2018$ & 2018 & $\begin{array}{l}\text { South Bay } \\
\text { (A5/A7 levee) }\end{array}$ & 2EEPSSB-A5A7 & 587481 & 4144081 & $06 / 07 / 2018$ & 51.71 & 60.81 & 39.71 \\
\hline SB-2 & Double-crested cormorant & $05 / 15 / 2018$ & 2018 & $\begin{array}{l}\text { South Bay } \\
\text { (A5/A7 levee) }\end{array}$ & 2EEPSSB-A5A7 & 587477 & 4144086 & $06 / 07 / 2018$ & 55.98 & 66.80 & 40.51 \\
\hline SB-3 & Double-crested cormorant & $05 / 15 / 2018$ & 2018 & $\begin{array}{l}\text { South Bay } \\
\text { (A5/A7 levee) }\end{array}$ & 2EEPSSB-A5A7 & 587470 & 4144093 & 06/07/2018 & 54.57 & 60.43 & 40.75 \\
\hline SB-4 & Double-crested cormorant & $05 / 15 / 2018$ & 2018 & $\begin{array}{l}\text { South Bay } \\
\text { (A5/A7 levee) }\end{array}$ & 2EEPSSB-A5A7 & 587471 & 4144092 & 06/07/2018 & 49.71 & 61.59 & 39.89 \\
\hline
\end{tabular}




\begin{tabular}{|c|c|c|c|c|c|c|c|c|c|c|c|}
\hline $\begin{array}{l}\text { USGS } \\
\text { egg ID }\end{array}$ & Species & $\begin{array}{c}\text { Collection } \\
\text { date } \\
\text { (mm/dd/yyyy) }\end{array}$ & Year & Location & Station code & $\begin{array}{c}\text { UTM- } \\
\text { easting } \\
\text { (NAD 83) }\end{array}$ & $\begin{array}{c}\text { UTM- } \\
\text { northing } \\
\text { (NAD 83) }\end{array}$ & $\begin{array}{c}\text { USGS egg } \\
\text { measuring date } \\
\text { (mm/dd/yyyy) }\end{array}$ & $\begin{array}{l}\text { USGS whole } \\
\text { egg mass } \\
\text { (g) }\end{array}$ & $\begin{array}{l}\text { USGS whole } \\
\text { egg length } \\
\text { (mm) }\end{array}$ & $\begin{array}{l}\text { USGS whole } \\
\text { egg width } \\
\text { (mm) }\end{array}$ \\
\hline SB-5 & Double-crested cormorant & $05 / 15 / 2018$ & 2018 & $\begin{array}{l}\text { South Bay } \\
\text { (A5/A7 levee) }\end{array}$ & 2EEPSSB-A5A7 & 587472 & 4144096 & $06 / 07 / 2018$ & 49.42 & 61.07 & 40.87 \\
\hline SB-6 & Double-crested cormorant & $05 / 15 / 2018$ & 2018 & $\begin{array}{l}\text { South Bay } \\
\text { (A5/A7 levee) }\end{array}$ & 2EEPSSB-A5A7 & 587476 & 4144087 & $06 / 07 / 2018$ & 49.16 & 61.78 & 39.67 \\
\hline SB-7 & Double-crested cormorant & $05 / 15 / 2018$ & 2018 & $\begin{array}{l}\text { South Bay } \\
\quad \text { (A5/A7 levee) }\end{array}$ & 2EEPSSB-A5A7 & 587428 & 4144123 & $06 / 08 / 2018$ & 42.21 & 60.94 & 37.24 \\
\hline SB-8 & Double-crested cormorant & $05 / 15 / 2018$ & 2018 & $\begin{array}{l}\text { South Bay } \\
\text { (A5/A7 levee) }\end{array}$ & 2EEPSSB-A5A7 & 587432 & 4144121 & $06 / 08 / 2018$ & 44.74 & 61.60 & 37.01 \\
\hline SB-9 & Double-crested cormorant & $05 / 15 / 2018$ & 2018 & $\begin{array}{l}\text { South Bay } \\
\quad \text { (A5/A7 levee) }\end{array}$ & 2EEPSSB-A5A7 & 587435 & 4144118 & $06 / 08 / 2018$ & 51.32 & 67.55 & 38.94 \\
\hline SB-10 & Double-crested cormorant & $05 / 15 / 2018$ & 2018 & $\begin{array}{l}\text { South Bay } \\
\quad \text { (A5/A7 levee) }\end{array}$ & 2EEPSSB-A5A7 & 587432 & 4144122 & $06 / 08 / 2018$ & 51.64 & 58.75 & 40.86 \\
\hline SB-11 & Double-crested cormorant & $05 / 15 / 2018$ & 2018 & $\begin{array}{l}\text { South Bay } \\
\quad \text { (A5/A7 levee) }\end{array}$ & 2EEPSSB-A5A7 & 587431 & 4144123 & $06 / 08 / 2018$ & 55.05 & 62.18 & 40.41 \\
\hline SB-12 & Double-crested cormorant & $05 / 15 / 2018$ & 2018 & $\begin{array}{l}\text { South Bay } \\
\quad \text { (A5/A7 levee) }\end{array}$ & 2EEPSSB-A5A7 & 587423 & 4144122 & $06 / 08 / 2018$ & 44.40 & 62.99 & 37.16 \\
\hline SB-13 & Double-crested cormorant & $05 / 15 / 2018$ & 2018 & $\begin{array}{l}\text { South Bay } \\
\quad \text { (A5/A7 levee) }\end{array}$ & 2EEPSSB-A5A7 & 587472 & 4144094 & $06 / 08 / 2018$ & 48.11 & 62.20 & 39.20 \\
\hline SB-14 & Double-crested cormorant & $05 / 15 / 2018$ & 2018 & $\begin{array}{l}\text { South Bay } \\
\text { (A5/A7 levee) }\end{array}$ & 2EEPSSB-A5A7 & 587465 & 4144093 & $06 / 08 / 2018$ & 37.78 & 55.73 & 36.66 \\
\hline SB-15 & Double-crested cormorant & $05 / 15 / 2018$ & 2018 & $\begin{array}{l}\text { South Bay } \\
\quad \text { (A5/A7 levee) }\end{array}$ & 2EEPSSB-A5A7 & 587473 & 4144093 & $06 / 08 / 2018$ & 51.04 & 61.13 & 41.81 \\
\hline SB-16 & Double-crested cormorant & $05 / 15 / 2018$ & 2018 & $\begin{array}{l}\text { South Bay } \\
\quad \text { (A5/A7 levee) }\end{array}$ & 2EEPSSB-A5A7 & 587464 & 4144096 & $06 / 08 / 2018$ & 45.42 & 60.54 & 38.74 \\
\hline SB-17 & Double-crested cormorant & $05 / 15 / 2018$ & 2018 & $\begin{array}{l}\text { South Bay } \\
\quad \text { (A5/A7 levee) }\end{array}$ & 2EEPSSB-A5A7 & 587466 & 4144097 & $06 / 08 / 2018$ & 47.72 & 59.91 & 39.46 \\
\hline SB-18 & Double-crested cormorant & $05 / 15 / 2018$ & 2018 & $\begin{array}{l}\text { South Bay } \\
\quad \text { (A5/A7 levee) }\end{array}$ & 2EEPSSB-A5A7 & 587475 & 4144092 & $06 / 08 / 2018$ & 43.33 & 61.24 & 37.72 \\
\hline SB-19 & Double-crested cormorant & $05 / 15 / 2018$ & 2018 & $\begin{array}{l}\text { South Bay } \\
\quad \text { (A5/A7 levee) }\end{array}$ & 2EEPSSB-A5A7 & 587431 & 4144120 & $06 / 08 / 2018$ & 45.78 & 60.90 & 37.22 \\
\hline SB-20 & Double-crested cormorant & $05 / 15 / 2018$ & 2018 & $\begin{array}{l}\text { South Bay } \\
\text { (A5/A7 levee) }\end{array}$ & 2EEPSSB-A5A7 & 587428 & 4144123 & $06 / 08 / 2018$ & 51.85 & 63.47 & 40.47 \\
\hline SB-21 & Double-crested cormorant & $05 / 15 / 2018$ & 2018 & $\begin{array}{l}\text { South Bay } \\
\quad \text { (A5/A7 levee) }\end{array}$ & 2EEPSSB-A5A7 & 587430 & 4144121 & $06 / 08 / 2018$ & 43.61 & 61.56 & 37.36 \\
\hline WI-1-N4 & Double-crested cormorant & 05/10/2018 & 2018 & Wheeler Island & 2EEPSWI & 590750 & 4215217 & $06 / 08 / 2018$ & 42.59 & 62.49 & 37.03 \\
\hline WI-2-N4 & Double-crested cormorant & $05 / 10 / 2018$ & 2018 & Wheeler Island & 2EEPSWI & 590750 & 4215217 & $06 / 08 / 2018$ & 44.74 & 65.21 & 36.66 \\
\hline WI-3-N4 & Double-crested cormorant & $05 / 10 / 2018$ & 2018 & Wheeler Island & 2EEPSWI & 590750 & 4215217 & $06 / 08 / 2018$ & 46.93 & 67.68 & 36.93 \\
\hline
\end{tabular}




\section{Appendix 2. Quality Assurance and Quality Control Results for Total Mercury (THg) Analyses of Forster's Tern Eggs Collected by U.S. Geological Survey (USGS) from San Francisco Bay, California, in 2018}

Table 2-1. Quality assurance and quality control: Certified reference materials.

[CRM, certified reference materials; mm/dd/yyyy, month/day/year; $\mu \mathrm{g} / \mathrm{g} \mathrm{dw}$, micrograms per gram dry weight; DORM-4, fish protein certified reference material for trace metals; THg, total mercury; TORT-3, lobster hepatopancreas reference material for trace metals]

\begin{tabular}{ccccc}
\hline $\begin{array}{c}\text { Analysis } \\
\text { date } \\
\text { (mm/dd/yyyy) }\end{array}$ & CRM & $\begin{array}{c}\text { Certified } \\
\text { value THg } \\
\text { ( } \mathbf{\mu g} / \mathbf{g ~ d w})\end{array}$ & $\begin{array}{c}\text { Measured } \\
\text { THg } \\
(\boldsymbol{\mu g} / \mathbf{g ~ d w})\end{array}$ & $\begin{array}{c}\text { Percent } \\
\text { recovery }\end{array}$ \\
\hline $09 / 18 / 2018$ & DORM-4 & 0.41 & 0.40 & 97.0 \\
$09 / 18 / 2018$ & DORM-4 & 0.41 & 0.41 & 100.3 \\
$09 / 19 / 2018$ & DORM-4 & 0.41 & 0.40 & 97.8 \\
$09 / 19 / 2018$ & DORM-4 & 0.41 & 0.41 & 100.1 \\
$09 / 19 / 2018$ & DORM-4 & 0.41 & 0.42 & 102.7 \\
$09 / 20 / 2018$ & DORM-4 & 0.41 & 0.40 & 98.7 \\
$09 / 20 / 2018$ & DORM-4 & 0.41 & 0.43 & 105.2 \\
$09 / 20 / 2018$ & DORM-4 & 0.41 & 0.42 & 101.9 \\
$09 / 21 / 2018$ & DORM-4 & 0.41 & 0.44 & 106.5 \\
$09 / 19 / 2018$ & TORT-3 & 0.29 & 0.30 & 102.0 \\
$09 / 19 / 2018$ & TORT-3 & 0.29 & 0.29 & 100.6 \\
$09 / 20 / 2018$ & TORT-3 & 0.29 & 0.30 & 101.6 \\
$09 / 21 / 2018$ & TORT-3 & 0.29 & 0.30 & 102.6 \\
\hline
\end{tabular}

${ }^{1}$ Percent recovery values are based on non-rounded data and therefore would differ slightly from the values obtained if they were calculated directly from the rounded data reported in these tables.

Table 2-2. Quality assurance and quality control: Continuing calibration verifications.

[CCV, continuing calibration verification; g, gram; mm/dd/yyyy, month/day/year; ng, nanogram; THg, total mercury; $\mu \mathrm{g} / \mathrm{g}$ ww, microgram per gram wet weight]

\begin{tabular}{|c|c|c|c|c|c|c|c|}
\hline $\begin{array}{c}\text { Analysis } \\
\text { date } \\
\text { (mm/dd/yyyy) }\end{array}$ & $\begin{array}{l}\text { CCV } \\
\text { (ng) }\end{array}$ & $\begin{array}{c}\text { CCV certified } \\
\text { value THg } \\
(\mu \mathrm{g} / \mathrm{g} \text { ww) }\end{array}$ & $\begin{array}{l}\text { Weight of } \\
\text { CCV sample } \\
\text { (g) }\end{array}$ & $\begin{array}{c}\text { Expected } \\
\text { THg } \\
\text { (ng) }\end{array}$ & $\begin{array}{l}\text { Measured } \\
\text { THg } \\
\text { (ng) }\end{array}$ & $\begin{array}{l}\text { Measured } \\
\text { THg } \\
(\mu \mathrm{g} / \mathrm{g} w w)\end{array}$ & $\begin{array}{l}\text { Percent } \\
\text { recovery }^{1}\end{array}$ \\
\hline $09 / 19 / 2018$ & 200 & 1.0170 & 0.19642 & 199.76 & 198.72200 & 1.01 & 99.5 \\
\hline 09/20/2018 & 200 & 1.0170 & 0.19671 & 200.05 & 197.26100 & 1.00 & 98.6 \\
\hline 09/21/2018 & 200 & 1.0170 & 0.19563 & 198.96 & 197.87900 & 1.01 & 99.5 \\
\hline 09/19/2018 & 50 & 1.0170 & 0.04850 & 49.32 & 49.34600 & 1.02 & 100.0 \\
\hline $09 / 21 / 2018$ & 50 & 1.0170 & 0.04933 & 50.17 & 50.41000 & 1.02 & 100.5 \\
\hline 09/18/2018 & 8.5 & 0.1060 & 0.08020 & 8.50 & 8.30400 & 0.10 & 97.7 \\
\hline 09/19/2018 & 8.5 & 0.1060 & 0.08059 & 8.54 & 8.77600 & 0.11 & 102.7 \\
\hline 09/19/2018 & 8.5 & 0.1060 & 0.08017 & 8.50 & 8.64100 & 0.11 & 101.7 \\
\hline
\end{tabular}

${ }^{1}$ Percent recovery values are based on non-rounded data and therefore would differ slightly from the values obtained if they were calculated directly from the rounded data reported in these tables. 
Table 2-3. Quality assurance and quality control: Sample duplicates.

[ID, identification; mm/dd/yyyy, month/day/year; THg, total mercury; $\mu \mathrm{g} / \mathrm{g}$ fww, microgram per gram fresh wet weight]

\begin{tabular}{|c|c|c|c|c|}
\hline $\begin{array}{c}\text { Analysis } \\
\text { date } \\
\text { (mm/dd/yyyy) }\end{array}$ & $\begin{array}{c}\text { Sample } \\
\text { ID }\end{array}$ & $\begin{array}{c}\mathrm{THg} \\
\text { ( } \mu \mathrm{g} / \mathrm{g} \text { fww) } \\
\text { replicate } 1\end{array}$ & $\begin{array}{c}\mathrm{THg} \\
\text { ( } \mu \mathrm{g} / \mathrm{g} \text { fww) } \\
\text { replicate } 2\end{array}$ & $\begin{array}{c}\text { Relative } \\
\text { percent } \\
\text { difference }^{1}\end{array}$ \\
\hline $09 / 18 / 2018$ & 18FE177 & 1.48 & 1.49 & 0.6 \\
\hline 09/19/2018 & 18FE119 & 1.34 & 1.34 & 0.6 \\
\hline 09/19/2018 & 18FE175 & 1.96 & 2.00 & 2.5 \\
\hline 09/19/2018 & 18FE183 & 2.44 & 2.39 & 1.8 \\
\hline $09 / 20 / 2018$ & 18FE172 & 1.90 & 1.97 & 3.4 \\
\hline $09 / 20 / 2018$ & 18FE23 & 0.81 & 0.80 & 1.2 \\
\hline $09 / 20 / 2018$ & 18 FE33 & 0.62 & 0.64 & 2.4 \\
\hline $09 / 21 / 2018$ & $18 \mathrm{FE} 21$ & 2.15 & 2.28 & 5.7 \\
\hline $09 / 21 / 2018$ & 18FE217 & 1.66 & 1.67 & 0.4 \\
\hline $09 / 21 / 2018$ & $18 \mathrm{FE} 3$ & 0.62 & 0.63 & 1.8 \\
\hline
\end{tabular}

'Relative percent difference values are based on non-rounded data and therefore would differ slightly from the values obtained if they were calculated directly from the rounded data reported in these tables.
Table 2-4. Quality assurance and quality control: System and method blanks.

[ID, identification; mm/dd/yyyy, month/day/year; $\mathrm{THg}$, total mercury; ng, nanogram]

\begin{tabular}{ccc}
\hline $\begin{array}{c}\text { Analysis date } \\
\text { (mm/dd/yyyy) }\end{array}$ & Sample ID & $\begin{array}{c}\text { THg } \\
(\mathbf{n g})\end{array}$ \\
\hline 09/18/2018 & System blank & 0.084 \\
09/18/2018 & Method blank & 0.085 \\
09/18/2018 & System blank & 0.229 \\
09/18/2018 & Method blank & 0.228 \\
09/18/2018 & System blank & 0.248 \\
09/18/2018 & Method blank & 0.252 \\
09/19/2018 & System blank & 0.211 \\
09/19/2018 & Method blank & 0.188 \\
09/19/2018 & System blank & 0.204 \\
09/19/2018 & Method blank & 0.195 \\
09/19/2018 & System blank & 0.22 \\
09/19/2018 & Method blank & 0.22 \\
09/20/2018 & System blank & 0.195 \\
09/20/2018 & Method blank & 0.203 \\
09/20/2018 & System blank & 0.219 \\
09/20/2018 & Method blank & 0.193 \\
09/20/2018 & System blank & 0.238 \\
09/20/2018 & Method blank & 0.232 \\
09/21/2018 & System blank & 0.263 \\
09/21/2018 & Method blank & 0.266 \\
\hline
\end{tabular}

Table 2-5. Quality assurance and quality control: Matrix spikes and matirx spike duplicates.

[g, gram; ID, identification; mm/dd/yyyy, month/day/year; ng, nanogram; THg, total mercury; $\mu \mathrm{g} / \mathrm{g}$ dw, microgram per gram dry weight]

\begin{tabular}{|c|c|c|c|c|c|c|c|c|c|}
\hline $\begin{array}{c}\text { Analysis } \\
\text { date } \\
\text { (mm/dd/ } \\
\text { yyyy) }\end{array}$ & Sample ID & $\begin{array}{l}\text { Weight of } \\
\text { egg } \\
\text { sample } \\
\text { (g) }\end{array}$ & $\begin{array}{c}\text { Mean THg } \\
\text { ( } \mu \mathrm{g} / \mathrm{g} \mathrm{dw}) \\
\text { of duplicate } \\
\text { samples prior } \\
\text { to spiking }\end{array}$ & $\begin{array}{c}\text { Expected } \\
\mathrm{THg} \\
(\mathrm{ng}) \\
\text { from egg } \\
\text { sample }\end{array}$ & $\begin{array}{c}\text { THg } \\
\text { (ng) } \\
\text { spike } \\
\text { amount }\end{array}$ & $\begin{array}{c}\text { Total expected } \\
\mathrm{THg} \\
\text { (ng) } \\
\text { of egg sample } \\
\text { + spike }\end{array}$ & $\begin{array}{l}\text { Measured } \\
\text { THg } \\
(\mathrm{ng}) \\
\text { egg sample } \\
\text { + spike }\end{array}$ & $\begin{array}{l}\text { Percent } \\
\text { recovered }\end{array}$ & $\begin{array}{l}\text { Relative percent } \\
\text { difference in } \\
\text { duplicate } \\
\text { spike samples }^{1}\end{array}$ \\
\hline $09 / 18 / 2018$ & 18FE177 & 0.02910 & 7.02 & 204.27 & 197.46 & 401.73 & 408.64 & 101.8 & 3.4 \\
\hline 09/18/2018 & 18FE177 & 0.03120 & 7.02 & 219.01 & 193.58 & 412.59 & 412.71 & 98.4 & 3.4 \\
\hline 09/19/2018 & 18FE119 & 0.03230 & 6.02 & 194.54 & 197.53 & 392.07 & 396.00 & 100.3 & 1.7 \\
\hline 09/19/2018 & 18FE119 & 0.03281 & 6.02 & 197.61 & 192.88 & 390.49 & 397.67 & 102.0 & 1.7 \\
\hline $09 / 20 / 2018$ & $18 \mathrm{FE} 172$ & 0.02747 & 8.68 & 238.41 & 198.65 & 437.06 & 437.89 & 98.7 & 0.7 \\
\hline 09/20/2018 & $18 \mathrm{FE} 172$ & 0.02792 & 8.68 & 242.31 & 199.40 & 441.71 & 441.20 & 98.1 & 0.7 \\
\hline $09 / 20 / 2018$ & $18 \mathrm{FE} 23$ & 0.02978 & 3.96 & 118.04 & 196.99 & 315.03 & 313.09 & 97.4 & 2.4 \\
\hline 09/20/2018 & 18FE23 & 0.02978 & 3.96 & 118.04 & 196.44 & 314.48 & 317.34 & 99.8 & 2.4 \\
\hline $09 / 21 / 2018$ & $18 \mathrm{FE} 3$ & 0.03305 & 2.98 & 98.54 & 198.53 & 297.07 & 303.76 & 101.6 & 0.0 \\
\hline $09 / 21 / 2018$ & $18 \mathrm{FE} 3$ & 0.03145 & 2.98 & 93.76 & 195.74 & 289.51 & 296.20 & 101.7 & 0.0 \\
\hline
\end{tabular}

${ }^{1}$ Relative percent difference values are based on non-rounded data and therefore would differ slightly from the values obtained if they were calculated directly from the rounded data reported in these tables. 
Appendix 3. Forster's Tern Egg Collection Data and Total Mercury (THg) Concentrations for Eggs Collected by USGS From San Francisco Bay, California, in 2018

[dw, dry weight; fww, fresh wet weight; g, gram; ID, identification; mm, millimeter; mm/dd/yyyy, month/day/year; NAD 83, North American Datum of 1983; $\mu \mathrm{g} / \mathrm{g}$, microgram per gram; ww, wet weight]

\begin{tabular}{|c|c|c|c|c|c|c|c|c|c|c|c|c|c|c|c|}
\hline $\begin{array}{c}\text { Sample } \\
\text { ID }\end{array}$ & $\begin{array}{c}\text { Composite } \\
\text { ID }\end{array}$ & $\begin{array}{c}\text { Egg } \\
\text { THg } \\
(\mu g / g d w)\end{array}$ & $\begin{array}{l}\text { Egg } \\
\mathrm{THg} \\
(\mu g / g \\
\mathrm{fww})\end{array}$ & Year & Colony & Station code & $\begin{array}{c}\text { Egg } \\
\text { collection } \\
\text { date } \\
\text { (mm/dd/yyyy) }\end{array}$ & $\begin{array}{l}\text { Whole } \\
\text { egg } \\
\text { mass } \\
(g)\end{array}$ & $\begin{array}{c}\text { Egg } \\
\text { length } \\
(\mathrm{mm})\end{array}$ & $\begin{array}{l}\text { Egg } \\
\text { width } \\
\text { (mm) }\end{array}$ & $\begin{array}{l}\text { UTM- } \\
\text { Easting } \\
\text { (NAD 83) }\end{array}$ & $\begin{array}{c}\text { UTM- } \\
\text { Northing } \\
\text { (NAD 83) }\end{array}$ & $\begin{array}{c}\text { Egg } \\
\text { content } \\
\text { mass } \\
(g \text { ww) }\end{array}$ & $\begin{array}{c}\text { Egg } \\
\text { content } \\
\text { mass } \\
(\mathbf{g ~ d w})\end{array}$ & $\begin{array}{l}\text { Percent } \\
\text { moisture }\end{array}$ \\
\hline 18FE171 & A3W-1 & 9.26 & 2.05 & 2018 & A3W & 2EEPSSB-A3W & $05 / 23 / 2018$ & 18.12 & 42.77 & 28.91 & 4143822 & 585791 & 16.53 & 3.92 & 76.3 \\
\hline 18FE175 & A3W-1 & 8.91 & 1.96 & 2018 & $\mathrm{~A} 3 \mathrm{~W}$ & 2EEPSSB-A3W & $05 / 23 / 2018$ & 18.28 & 47.03 & 30.02 & 4143832 & 585788 & 16.61 & 4.62 & 72.2 \\
\hline 18FE182 & A3W-1 & 7.00 & 1.50 & 2018 & $\mathrm{~A} 3 \mathrm{~W}$ & 2EEPSSB-A3W & $05 / 23 / 2018$ & 21.30 & 47.24 & 30.36 & 4143833 & 585790 & 19.57 & 4.65 & 76.3 \\
\hline 18FE87 & A3W-1 & 5.18 & 1.10 & 2018 & A3W & 2EEPSSB-A3W & 05/23/2018 & 19.93 & 44.80 & 31.20 & 4143830 & 585789 & 18.14 & 4.60 & 74.6 \\
\hline 18FE88 & A3W-1 & 8.05 & 1.73 & 2018 & $\mathrm{~A} 3 \mathrm{~W}$ & 2EEPSSB-A3W & $05 / 23 / 2018$ & 18.16 & 43.04 & 30.76 & 4143277 & 585586 & 16.62 & 4.37 & 73.7 \\
\hline $18 \mathrm{FE} 89$ & A3W-1 & 11.55 & 2.49 & 2018 & $\mathrm{~A} 3 \mathrm{~W}$ & 2EEPSSB-A3W & 05/23/2018 & 18.26 & 41.89 & 29.40 & 4143277 & 585583 & 16.83 & 3.89 & 76.9 \\
\hline 18FE98 & A3W-1 & 4.38 & 0.96 & 2018 & $\mathrm{~A} 3 \mathrm{~W}$ & 2EEPSSB-A3W & $05 / 23 / 2018$ & 18.86 & 42.18 & 30.11 & 4143279 & 585584 & 17.26 & 4.15 & 75.9 \\
\hline 18FE178 & A3W-2 & 5.29 & 1.19 & 2018 & $\mathrm{~A} 3 \mathrm{~W}$ & 2EEPSSB-A3W & 05/23/2018 & 18.98 & 43.50 & 29.80 & 4143828 & 585790 & 17.45 & 4.34 & 75.1 \\
\hline 18FE181 & A3W-2 & 10.03 & 2.10 & 2018 & $\mathrm{~A} 3 \mathrm{~W}$ & 2EEPSSB-A3W & $05 / 23 / 2018$ & 20.08 & 46.10 & 30.22 & 4143833 & 585790 & 18.41 & 4.39 & 76.2 \\
\hline 18FE184 & A3W-2 & 6.86 & 1.41 & 2018 & A3W & 2EEPSSB-A3W & $05 / 23 / 2018$ & 18.28 & 43.74 & 30.70 & 4143832 & 585790 & 16.70 & 4.22 & 74.7 \\
\hline 18FE29 & A3W-2 & 8.99 & 1.90 & 2018 & $\mathrm{~A} 3 \mathrm{~W}$ & 2EEPSSB-A3W & 05/23/2018 & 18.86 & 43.11 & 30.89 & 4143277 & 585586 & 17.27 & 4.34 & 74.9 \\
\hline 18FE90 & $\mathrm{A} 3 \mathrm{~W}-2$ & 6.66 & 1.35 & 2018 & $\mathrm{~A} 3 \mathrm{~W}$ & 2EEPSSB-A3W & 05/23/2018 & 18.41 & 44.57 & 30.32 & 4143831 & 585790 & 16.79 & 4.14 & 75.3 \\
\hline 18FE92 & A3W-2 & 14.33 & 2.97 & 2018 & A3W & 2EEPSSB-A3W & $05 / 23 / 2018$ & 18.89 & 43.60 & 31.16 & 4143830 & 585780 & 17.42 & 4.40 & 74.8 \\
\hline 18FE95 & A3W-2 & 5.12 & 1.23 & 2018 & $\mathrm{~A} 3 \mathrm{~W}$ & 2EEPSSB-A3W & 05/23/2018 & 18.24 & 38.18 & 30.42 & 4143527 & 585712 & 16.47 & 4.20 & 74.5 \\
\hline 18FE177 & A3W-3 & 7.00 & 1.48 & 2018 & $\mathrm{~A} 3 \mathrm{~W}$ & 2EEPSSB-A3W & $05 / 23 / 2018$ & 20.54 & 45.41 & 30.76 & 4143276 & 585583 & 18.92 & 4.53 & 76.0 \\
\hline 18FE183 & A3W-3 & 11.47 & 2.44 & 2018 & $\mathrm{~A} 3 \mathrm{~W}$ & 2EEPSSB-A3W & $05 / 23 / 2018$ & 17.90 & 43.85 & 29.05 & 4143525 & 585714 & 16.43 & 3.92 & 76.2 \\
\hline $18 \mathrm{FE} 226$ & A3W-3 & 5.49 & 1.24 & 2018 & A3W & 2EEPSSB-A3W & 06/01/2018 & 17.83 & 43.28 & 29.76 & 4143276 & 585583 & 16.21 & 4.31 & 73.4 \\
\hline 18FE31 & A3W-3 & 10.37 & 2.30 & 2018 & $\mathrm{~A} 3 \mathrm{~W}$ & 2EEPSSB-A3W & $05 / 23 / 2018$ & 19.40 & 42.80 & 30.48 & 4143276 & 585583 & 17.85 & 4.39 & 75.4 \\
\hline $18 \mathrm{FE} 5$ & A3W-3 & 4.87 & 1.10 & 2018 & $\mathrm{~A} 3 \mathrm{~W}$ & 2EEPSSB-A3W & $05 / 23 / 2018$ & 21.67 & 47.58 & 30.24 & 4143831 & 585791 & 19.99 & 4.87 & 75.6 \\
\hline 18FE86 & A3W-3 & 5.80 & 1.25 & 2018 & A3W & 2EEPSSB-A3W & $05 / 23 / 2018$ & 18.13 & 42.76 & 30.32 & 4143831 & 585893 & 16.49 & 4.20 & 74.5 \\
\hline 18FE91 & A3W-3 & 11.90 & 2.56 & 2018 & $\mathrm{~A} 3 \mathrm{~W}$ & 2EEPSSB-A3W & $05 / 23 / 2018$ & 18.76 & 45.33 & 29.70 & 4143524 & 585714 & 17.06 & 4.27 & 75.0 \\
\hline $18 \mathrm{FE} 100$ & N1-1 & 2.30 & 0.53 & 2018 & N1 & 2EEPSSB-N1 & $05 / 25 / 2018$ & 17.59 & 40.55 & 29.90 & 4153620 & 581043 & 16.08 & 4.13 & 74.3 \\
\hline $18 \mathrm{FE} 172$ & N1-1 & 8.53 & 1.90 & 2018 & N1 & 2EEPSSB-N1 & $05 / 25 / 2018$ & 17.76 & 43.65 & 30.67 & 4153660 & 581064 & 16.18 & 4.57 & 71.7 \\
\hline 18FE187 & N1-1 & 7.32 & 1.59 & 2018 & N1 & 2EEPSSB-N1 & $06 / 13 / 2018$ & 18.63 & 45.24 & 29.25 & 4153552 & 581413 & 17.17 & 4.20 & 75.5 \\
\hline 18FE3 & N1-1 & 2.95 & 0.62 & 2018 & N1 & 2EEPSSB-N1 & 05/18/2018 & 19.81 & 42.97 & 30.38 & 4153623 & 581045 & 18.20 & 4.15 & 77.2 \\
\hline $18 \mathrm{FE} 32$ & N1-1 & 4.03 & 0.88 & 2018 & N1 & 2EEPSSB-N1 & 05/18/2018 & 20.89 & 44.66 & 31.22 & 4153623 & 581045 & 19.19 & 4.76 & 75.2 \\
\hline 18FE94 & N1-1 & 4.87 & 1.04 & 2018 & N1 & 2EEPSSB-N1 & $05 / 25 / 2018$ & 18.72 & 44.17 & 30.61 & 4153620 & 581053 & 17.26 & 4.43 & 74.3 \\
\hline 18FE97 & N1-1 & 7.27 & 1.57 & 2018 & N1 & 2EEPSSB-N1 & $05 / 25 / 2018$ & 19.44 & 44.11 & 31.29 & 4153620 & 581043 & 17.75 & 4.65 & 73.8 \\
\hline
\end{tabular}




\begin{tabular}{|c|c|c|c|c|c|c|c|c|c|c|c|c|c|c|c|}
\hline $\begin{array}{c}\text { Sample } \\
\text { ID }\end{array}$ & $\begin{array}{c}\text { Composite } \\
\text { ID }\end{array}$ & $\begin{array}{c}\text { Egg } \\
\text { THg } \\
(\mu \mathrm{g} / \mathrm{g} \mathrm{dw})\end{array}$ & $\begin{array}{l}\text { Egg } \\
\mathrm{THg} \\
(\mu \mathrm{g} / \mathrm{g} \\
\mathrm{fww})\end{array}$ & Year & Colony & Station code & $\begin{array}{c}\text { Egg } \\
\text { collection } \\
\text { date } \\
\text { (mm/dd/yyyy) }\end{array}$ & $\begin{array}{l}\text { Whole } \\
\text { egg } \\
\text { mass } \\
(g)\end{array}$ & $\begin{array}{c}\text { Egg } \\
\text { length } \\
(\mathrm{mm})\end{array}$ & $\begin{array}{c}\text { Egg } \\
\text { width } \\
(\mathrm{mm})\end{array}$ & $\begin{array}{c}\text { UTM- } \\
\text { Easting } \\
\text { (NAD 83) }\end{array}$ & $\begin{array}{c}\text { UTM- } \\
\text { Northing } \\
\text { (NAD 83) }\end{array}$ & $\begin{array}{c}\text { Egg } \\
\text { content } \\
\text { mass } \\
(g \text { ww) }\end{array}$ & $\begin{array}{c}\text { Egg } \\
\text { content } \\
\text { mass } \\
(\mathrm{g} \mathrm{dw})\end{array}$ & $\begin{array}{l}\text { Percent } \\
\text { moisture }\end{array}$ \\
\hline $18 \mathrm{FE} 174$ & $\mathrm{~N} 1-2$ & 4.62 & 1.01 & 2018 & N1 & 2EEPSSB-N1 & $05 / 25 / 2018$ & 19.78 & 43.59 & 30.82 & 4153623 & 581045 & 18.21 & 4.52 & 75.2 \\
\hline $18 \mathrm{FE} 176$ & N1-2 & 5.34 & 1.03 & 2018 & N1 & 2EEPSSB-N1 & $05 / 25 / 2018$ & 20.99 & 46.62 & 30.81 & 4153623 & 581046 & 19.36 & 4.28 & 77.9 \\
\hline 18FE198 & N1-2 & 3.92 & 0.86 & 2018 & N1 & 2EEPSSB-N1 & 06/13/2018 & 17.97 & 41.20 & 29.78 & 4153549 & 581412 & 16.47 & 3.97 & 75.9 \\
\hline 18FE2 & N1-2 & 3.01 & 0.64 & 2018 & N1 & 2EEPSSB-N1 & 05/18/2018 & 18.43 & 43.36 & 30.75 & 4153622 & 581045 & 16.76 & 4.33 & 74.2 \\
\hline 18 FE220 & N1-2 & 10.64 & 1.99 & 2018 & N1 & 2EEPSSB-N1 & $06 / 13 / 2018$ & 18.76 & 43.34 & 31.32 & 4153550 & 581412 & 17.16 & 3.95 & 77.0 \\
\hline 18FE93 & N1-2 & 8.63 & 1.81 & 2018 & N1 & 2EEPSSB-N1 & $06 / 13 / 2018$ & 17.91 & 42.50 & 29.64 & 4158540 & 581411 & 16.31 & 3.88 & 76.2 \\
\hline 18FE96 & N1-2 & 7.31 & 1.55 & 2018 & N1 & 2EEPSSB-N1 & $05 / 25 / 2018$ & 19.94 & 43.34 & 31.48 & 4153620 & 581044 & 18.43 & 4.57 & 75.2 \\
\hline 18FE136 & $\mathrm{N} 1-3$ & 4.95 & 1.06 & 2018 & N1 & 2EEPSSB-N1 & 07/17/2018 & 16.63 & 41.97 & 28.95 & 4153624 & 581045 & 15.06 & 3.73 & 75.2 \\
\hline 18FE179 & $\mathrm{N} 1-3$ & 4.56 & 1.00 & 2018 & $\mathrm{~N} 1$ & 2EEPSSB-N1 & $05 / 25 / 2018$ & 18.09 & 44.31 & 29.91 & 4153621 & 581045 & 16.62 & 4.35 & 73.8 \\
\hline 18FE185 & $\mathrm{N} 1-3$ & 6.18 & 1.31 & 2018 & N1 & 2EEPSSB-N1 & $05 / 25 / 2018$ & 16.89 & 42.15 & 29.19 & 4153621 & 583048 & 15.47 & 3.80 & 75.4 \\
\hline 18FE209 & N1-3 & 5.80 & 1.23 & 2018 & N1 & 2EEPSSB-N1 & $06 / 13 / 2018$ & 21.10 & 44.05 & 31.51 & 4153541 & 581412 & 19.46 & 4.65 & 76.1 \\
\hline 18FE22 & $\mathrm{N} 1-3$ & 3.51 & 0.74 & 2018 & N1 & 2EEPSSB-N1 & 05/30/2018 & 20.15 & 45.64 & 30.56 & 4153622 & 581044 & 18.44 & 4.49 & 75.7 \\
\hline 18FE35 & N1-3 & 2.25 & 0.51 & 2018 & N1 & 2EEPSSB-N1 & 06/13/2018 & 17.92 & 40.75 & 30.05 & 4153538 & 581409 & 16.49 & 4.13 & 75.0 \\
\hline 18FE37 & $\mathrm{N} 1-3$ & 5.22 & 1.22 & 2018 & N1 & 2EEPSSB-N1 & $06 / 13 / 2018$ & 19.00 & 40.85 & 30.59 & 4153538 & 581411 & 17.47 & 4.46 & 74.5 \\
\hline 18FE1 & NCM-1 & 6.88 & 1.46 & 2018 & $\mathrm{NCM}$ & 2EEPSSB-NCM & $05 / 22 / 2018$ & 18.72 & 42.03 & 30.31 & 4143803 & 591486 & 17.21 & 4.10 & 76.2 \\
\hline 18FE10 & NCM-1 & 1.23 & 0.30 & 2018 & $\mathrm{NCM}$ & 2EEPSSB-NCM & 05/17/2018 & 17.64 & 42.05 & 30.32 & 4143756 & 591505 & 15.80 & 4.71 & 70.2 \\
\hline 18FE26 & NCM-1 & 8.11 & 1.84 & 2018 & $\mathrm{NCM}$ & 2EEPSSB-NCM & $05 / 22 / 2018$ & 18.83 & 45.71 & 29.76 & 4143792 & 591503 & 17.40 & 4.61 & 73.5 \\
\hline $18 \mathrm{FE} 27$ & NCM-1 & 1.99 & 0.50 & 2018 & $\mathrm{NCM}$ & 2EEPSSB-NCM & $05 / 22 / 2018$ & 18.12 & 44.85 & 29.60 & 4144081 & 590918 & 16.39 & 4.89 & 70.2 \\
\hline 18FE30 & NCM-1 & 11.76 & 2.48 & 2018 & $\mathrm{NCM}$ & 2EEPSSB-NCM & $05 / 22 / 2018$ & 19.09 & 41.12 & 30.79 & 4144110 & 590919 & 17.51 & 4.11 & 76.6 \\
\hline 18FE6 & NCM-1 & 5.33 & 1.15 & 2018 & $\mathrm{NCM}$ & 2EEPSSB-NCM & 05/17/2018 & 20.72 & 45.59 & 30.66 & 4143801 & 591495 & 19.20 & 4.62 & 75.9 \\
\hline 18 FE9 & NCM-1 & 2.40 & 0.52 & 2018 & $\mathrm{NCM}$ & 2EEPSSB-NCM & $05 / 17 / 2018$ & 20.05 & 43.31 & 30.72 & 4143800 & 591522 & 18.36 & 4.39 & 76.1 \\
\hline 18FE11 & NCM-2 & 6.76 & 1.57 & 2018 & $\mathrm{NCM}$ & 2EEPSSB-NCM & 05/17/2018 & 21.76 & 43.01 & 31.94 & 4143778 & 591502 & 20.18 & 5.08 & 74.8 \\
\hline 18FE13 & NCM-2 & 9.55 & 2.06 & 2018 & $\mathrm{NCM}$ & 2EEPSSB-NCM & $05 / 17 / 2018$ & 18.92 & 42.92 & 30.41 & 4143805 & 591536 & 17.38 & 4.28 & 75.4 \\
\hline 18 FE18 & NCM-2 & 5.34 & 1.03 & 2018 & $\mathrm{NCM}$ & 2EEPSSB-NCM & 05/17/2018 & 19.79 & 43.39 & 31.04 & 4143808 & 591508 & 18.01 & 4.01 & 77.7 \\
\hline 18FE208 & NCM-2 & 5.37 & 1.20 & 2018 & $\mathrm{NCM}$ & 2EEPSSB-NCM & 06/07/2018 & 19.32 & 43.48 & 30.59 & 4144105 & 590976 & 17.46 & 4.51 & 74.2 \\
\hline 18FE25 & NCM-2 & 11.94 & 2.55 & 2018 & $\mathrm{NCM}$ & 2EEPSSB-NCM & $05 / 22 / 2018$ & 17.30 & 42.82 & 29.87 & 4143806 & 591519 & 15.87 & 4.08 & 74.3 \\
\hline $18 \mathrm{FE} 40$ & NCM-2 & 9.53 & 2.03 & 2018 & $\mathrm{NCM}$ & 2EEPSSB-NCM & $05 / 22 / 2018$ & 18.61 & 44.20 & 30.85 & 4143814 & 591530 & 17.13 & 4.48 & 73.8 \\
\hline 18FE7 & NCM-2 & 2.62 & 0.59 & 2018 & $\mathrm{NCM}$ & 2EEPSSB-NCM & 05/17/2018 & 18.80 & 42.21 & 30.61 & 4143792 & 591536 & 17.04 & 4.40 & 74.2 \\
\hline 18FE119 & NCM-3 & 6.00 & 1.34 & 2018 & $\mathrm{NCM}$ & 2EEPSSB-NCM & $07 / 10 / 2018$ & 17.95 & 43.04 & 29.89 & 4144100 & 590975 & 16.31 & 4.25 & 74.0 \\
\hline 18FE12 & NCM-3 & 6.30 & 1.42 & 2018 & $\mathrm{NCM}$ & 2EEPSSB-NCM & 05/17/2018 & 19.86 & 43.72 & 30.55 & 4144121 & 590981 & 18.18 & 4.57 & 74.9 \\
\hline $18 \mathrm{FE} 145$ & NCM-3 & 1.26 & 0.30 & 2018 & $\mathrm{NCM}$ & 2EEPSSB-NCM & 07/10/2018 & 18.95 & 44.44 & 31.05 & 4144102 & 590977 & 17.19 & 5.16 & 70.0 \\
\hline $18 \mathrm{FE} 16$ & NCM-3 & 16.91 & 3.60 & 2018 & $\mathrm{NCM}$ & 2EEPSSB-NCM & $05 / 22 / 2018$ & 17.67 & 43.99 & 29.78 & 4144109 & 590978 & 15.97 & 4.12 & 74.2 \\
\hline
\end{tabular}




\begin{tabular}{|c|c|c|c|c|c|c|c|c|c|c|c|c|c|c|c|}
\hline $\begin{array}{c}\text { Sample } \\
\text { ID }\end{array}$ & $\begin{array}{c}\text { Composite } \\
\text { ID }\end{array}$ & $\begin{array}{c}\text { Egg } \\
\text { THg } \\
(\mu \mathrm{g} / \mathrm{g} \mathrm{dw})\end{array}$ & $\begin{array}{l}\text { Egg } \\
\mathrm{THg} \\
(\mu \mathrm{g} / \mathrm{g} \\
\mathrm{fww})\end{array}$ & Year & Colony & Station code & $\begin{array}{c}\text { Egg } \\
\text { collection } \\
\text { date } \\
\text { (mm/dd/yyyy) }\end{array}$ & $\begin{array}{l}\text { Whole } \\
\text { egg } \\
\text { mass } \\
(g)\end{array}$ & $\begin{array}{c}\text { Egg } \\
\text { length } \\
(\mathrm{mm})\end{array}$ & $\begin{array}{c}\text { Egg } \\
\text { width } \\
\text { (mm) }\end{array}$ & $\begin{array}{c}\text { UTM- } \\
\text { Easting } \\
\text { (NAD 83) }\end{array}$ & $\begin{array}{c}\text { UTM- } \\
\text { Northing } \\
\text { (NAD 83) }\end{array}$ & $\begin{array}{c}\text { Egg } \\
\text { content } \\
\text { mass } \\
\text { (g ww) }\end{array}$ & $\begin{array}{c}\text { Egg } \\
\text { content } \\
\text { mass } \\
(\mathrm{g} \mathrm{dw})\end{array}$ & $\begin{array}{l}\text { Percent } \\
\text { moisture }\end{array}$ \\
\hline 18FE28 & NCM-3 & 14.37 & 3.14 & 2018 & $\mathrm{NCM}$ & 2EEPSSB-NCM & $05 / 22 / 2018$ & 16.48 & 41.77 & 29.11 & 4143799 & 591530 & 15.05 & 3.86 & 74.4 \\
\hline 18FE39 & NCM-3 & 3.95 & 0.87 & 2018 & $\mathrm{NCM}$ & 2EEPSSB-NCM & $05 / 22 / 2018$ & 20.31 & 45.24 & 30.92 & 4143822 & 591514 & 18.72 & 4.76 & 74.6 \\
\hline $18 \mathrm{FE} 8$ & NCM-3 & 9.20 & 1.92 & 2018 & $\mathrm{NCM}$ & 2EEPSSB-NCM & 05/17/2018 & 18.59 & 44.45 & 30.43 & 4143787 & 591532 & 16.93 & 4.28 & 74.7 \\
\hline 18FE173 & SF2-1 & 1.90 & 0.41 & 2018 & SF2 & 2EEPSSB-SF2 & $05 / 31 / 2018$ & 19.18 & 42.57 & 30.27 & 4150336 & 576952 & 17.56 & 4.18 & 76.2 \\
\hline 18FE188 & SF2-1 & 10.56 & 2.25 & 2018 & SF2 & 2EEPSSB-SF2 & $06 / 12 / 2018$ & 18.94 & 44.10 & 29.87 & 4150249 & 576855 & 17.30 & 4.17 & 75.9 \\
\hline 18FE201 & SF2-1 & 11.31 & 2.47 & 2018 & $\mathrm{SF} 2$ & 2EEPSSB-SF2 & $06 / 12 / 2018$ & 20.31 & 44.95 & 30.80 & 4150339 & 576954 & 18.64 & 4.63 & 75.1 \\
\hline 18FE211 & SF2-1 & 8.64 & 1.92 & 2018 & SF2 & 2EEPSSB-SF2 & 06/05/2018 & 17.45 & 41.68 & 29.11 & 4150337 & 576950 & 15.99 & 3.90 & 75.6 \\
\hline 18FE23 & SF2-1 & 3.99 & 0.81 & 2018 & SF2 & 2EEPSSB-SF2 & $05 / 31 / 2018$ & 18.09 & 42.67 & 30.06 & 4150252 & 576856 & 16.30 & 3.88 & 76.2 \\
\hline 18FE235 & SF2-1 & 10.01 & 2.25 & 2018 & SF2 & 2EEPSSB-SF2 & $05 / 31 / 2018$ & 20.98 & 44.53 & 30.71 & 4149782 & 576938 & 19.28 & 4.69 & 75.7 \\
\hline 18FE99 & SF2-1 & 2.52 & 0.55 & 2018 & SF2 & 2EEPSSB-SF2 & $06 / 12 / 2018$ & 18.49 & 43.25 & 29.77 & 4150346 & 576988 & 17.11 & 4.21 & 75.4 \\
\hline 18FE186 & SF2-2 & 6.17 & 1.41 & 2018 & SF2 & 2EEPSSB-SF2 & $06 / 12 / 2018$ & 16.79 & 42.83 & 28.49 & 4150350 & 576942 & 15.41 & 3.95 & 74.4 \\
\hline 18FE202 & SF2-2 & 11.47 & 2.39 & 2018 & SF2 & 2EEPSSB-SF2 & 06/05/2018 & 19.42 & 42.33 & 31.33 & 4150073 & 576930 & 17.78 & 4.33 & 75.7 \\
\hline 18FE222 & SF2-2 & 8.40 & 1.91 & 2018 & SF2 & 2EEPSSB-SF2 & 06/05/2018 & 21.20 & 43.44 & 30.91 & 4150206 & 576957 & 19.37 & 4.67 & 75.9 \\
\hline 18FE225 & SF2-2 & 7.26 & 1.57 & 2018 & SF2 & 2EEPSSB-SF2 & 06/05/2018 & 16.54 & 42.18 & 29.01 & 4150252 & 576856 & 15.09 & 3.82 & 74.7 \\
\hline 18FE231 & SF2-2 & 4.54 & 0.99 & 2018 & SF2 & 2EEPSSB-SF2 & $05 / 31 / 2018$ & 18.13 & 42.89 & 29.22 & 4149777 & 576938 & 16.67 & 3.97 & 76.2 \\
\hline 18FE24 & SF2-2 & 5.26 & 1.06 & 2018 & SF2 & 2EEPSSB-SF2 & 06/12/2018 & 20.19 & 43.94 & 31.17 & 4150249 & 576857 & 18.38 & 4.26 & 76.8 \\
\hline 18FE33 & SF2-2 & 3.02 & 0.62 & 2018 & SF2 & 2EEPSSB-SF2 & $06 / 12 / 2018$ & 20.48 & 46.60 & 30.30 & 4150342 & 576995 & 19.02 & 4.41 & 76.8 \\
\hline 18FE197 & SF2-3 & 10.96 & 2.36 & 2018 & SF2 & 2EEPSSB-SF2 & $06 / 12 / 2018$ & 19.44 & 42.82 & 30.78 & 4150340 & 576954 & 17.73 & 4.35 & 75.5 \\
\hline 18FE203 & SF2-3 & 3.70 & 0.77 & 2018 & SF2 & 2EEPSSB-SF2 & $06 / 05 / 2018$ & 19.61 & 44.19 & 29.94 & 4150130 & 576965 & 18.10 & 4.12 & 77.2 \\
\hline 18FE206 & SF2-3 & 7.54 & 1.61 & 2018 & SF2 & 2EEPSSB-SF2 & $06 / 12 / 2018$ & 17.99 & 41.53 & 29.90 & 4150248 & 576856 & 16.43 & 3.95 & 76.0 \\
\hline 18FE21 & SF2-3 & 9.79 & 2.15 & 2018 & SF2 & 2EEPSSB-SF2 & $05 / 24 / 2018$ & 15.63 & 39.24 & 28.53 & 4150250 & 576857 & 14.28 & 3.49 & 75.5 \\
\hline 18 FE228 & SF2-3 & 3.63 & 0.75 & 2018 & SF2 & 2EEPSSB-SF2 & $06 / 05 / 2018$ & 18.77 & 43.28 & 30.38 & 4150246 & 576856 & 17.28 & 4.11 & 76.2 \\
\hline 18FE229 & SF2-3 & 6.35 & 1.36 & 2018 & SF2 & 2EEPSSB-SF2 & $06 / 05 / 2018$ & 20.21 & 42.94 & 30.76 & 4150252 & 576856 & 18.53 & 4.34 & 76.6 \\
\hline 18 FE230 & SF2-3 & 5.92 & 1.28 & 2018 & SF2 & 2EEPSSB-SF2 & $06 / 05 / 2018$ & 21.00 & 43.25 & 31.72 & 4150252 & 576856 & 19.42 & 4.70 & 75.8 \\
\hline
\end{tabular}


For more information concerning the research in this report, contact the Director, Western Ecological Research Center

U.S. Geological Survey

3020 State University Drive East

Sacramento, California 95819

https://www.usgs.gov/centers/werc 


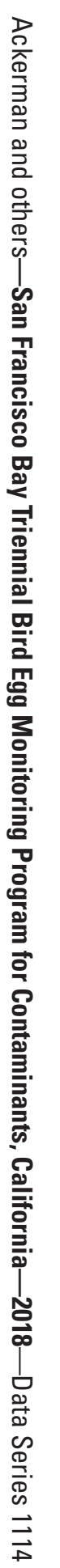

